\section{Combatting infection risk in veterinary practice through novel animation methods}

\section{Fraje Watson ${ }^{1,2}$, Matthieu Poyade ${ }^{3}$, Shona Noble ${ }^{3}$, Mark Chambers ${ }^{4}$, Roberto La Ragione ${ }^{4}$, Kayleigh Wyles ${ }^{4}$, Andrew Wales ${ }^{4}$, Tom Kupfer ${ }^{5}$, Alastair Macdonald ${ }^{3}$}

\footnotetext{
1 University College London, London, United Kingdom

2 Fitzpatrick Referrals, Godalming, United Kingdom

3 Glasgow School of Art, Glasgow, United Kingdom

4 University of Surrey, Guildford, United Kingdom

5 Vrije Universiteit Amsterdam, Amsterdam, Netherlands
}

\section{OBJECTIVES}

To address antimicrobial resistance by assisting veterinary practice staff to visualise bacterial cross-contamination and its control via an engaging three-dimensional graphical simulation tool. To develop and deploy the tool and measure its effects upon the perceptions of veterinary staff about contamination and infection risk.

\section{METHODS}

Workflow and orthopaedic procedures were filmed at a referral practice, informing the selection and modelling of a pre-surgical preparation sequence for the graphical tool. Further refinement and the mode of use emerged from workshops involving clinicians from a UK Veterinary School

The tool was deployed at the referral practice over 3 days in structured, interactive workshops. Before this, and shortly afterwards, test and control groups of staff completed questionnaires regarding perceptions of infection prevention and control.

\section{RESULTS}

The simulation that emerged was a monochrome surgical preparation area with clinical staff and a canine patient. Colour-coding highlighted infection prevention measures (green), which interacted with pink areas of bacterial contamination. The sequence was short, allowing repeated runs whilst changing elements to show effects of control measures on contamination spread. Workshop participants (51) enjoyed the approach; comments included: "since doing the training, whenever I do anything now, all I see is red [the invisible is now visible!]" and "this demonstration would be extremely beneficial for the entire team throughout the practice".

\section{STATEMENT (CONCLUSIONS)}

Analysis of questionnaire data is in progress and quantitative results will be presented. The approach has substantial potential to be deployed in other modes also, particularly self-learning via web and tablet applications. 